\title{
Medication Management Frameworks in the Context of Self-Management: A Scoping Review
}

\author{
Lauren Cadel (iD ${ }^{1,2}$ \\ Stephanie R Cimino ${ }^{3,4}$ \\ Teagan Rolf von den Baumen (iD) \\ Kadesha A James' \\ Lisa McCarthy ${ }^{1,2,5}$ \\ Sara JT Guilcher (D) 1,3,6 \\ 'Leslie Dan Faculty of Pharmacy, \\ University of Toronto, Toronto, Ontario, \\ Canada; ${ }^{2}$ Institute for Better Health, \\ Trillium Health Partners, Mississauga, \\ Ontario, Canada; ${ }^{3}$ Rehabilitation Sciences \\ Institute, University of Toronto, Toronto, \\ Ontario, Canada; ${ }^{4}$ St. John's Rehab, \\ Sunnybrook Health Sciences Centre, \\ North York, Ontario, Canada; ${ }^{5}$ Women's \\ College Research Institute, Women's \\ College Hospital, Toronto, Ontario, \\ Canada; ' Institute of Health Policy, \\ Management and Evaluation, University of \\ Toronto, Toronto, Ontario, Canada
}

Correspondence: Sara JT Guilcher

Leslie Dan Faculty of Pharmacy, 144

College Street, Room 604, Toronto, ON,

M5S 3M2, Canada

Tel + I-4I6-946-7020

Email sara.guilcher@utoronto.ca
Purpose: Many individuals take multiple prescribed and unprescribed medications, also known as polypharmacy, which can be problematic. Improving medication self-management is important; however, most medication management frameworks focus on adherence and limit the integration of the core components of self-management. Therefore, the objective of this scoping review was to identify what is reported in the literature on medication management frameworks or models within the context of self-management.

Methods: Electronic databases (Medline, Embase, CINAHL and Cochrane Library) and grey literature (healthcare and government organization websites) were searched for articles that described a framework or model developed or adapted for medication management, included components of self-management and was published from January 2000 to January 2020. During the screening of titles and abstracts, 5668 articles were reviewed, 5242 were excluded and 426 were then assessed at the full-text level. Thirty-nine articles met the eligibility criteria and were included in the review.

Results: About half of the frameworks were newly developed $(n=20)$, while the other half were adapted from, or applied, a previous model or framework $(n=19)$. The majority of frameworks focused on medication adherence and most of the self-management domains were categorized as medical management, followed by emotional and role management.

Conclusion: Medication self-management is a complex process and often impacts multiple areas of an individual's life. It is important for future frameworks to incorporate a comprehensive, holistic conceptualization of self-management that is inclusive of the three selfmanagement domains - medical, emotional and role management.

Keywords: medication therapy management, self-management, framework, model, review

\section{Introduction}

The use of medication, prescribed and unprescribed, is common for the management of both acute and chronic health conditions, as well as for the maintenance of overall health. ${ }^{1,2}$ There is a high prevalence of prescription medication use worldwide, with some countries reporting an increase in medication use over the last 20 years. ${ }^{3-5}$ Among individuals taking medication, there is a large portion who are taking multiple medications (ie, polypharmacy). Polypharmacy is most commonly defined as the concomitant use of five or more medications, which often includes prescription medications, over-thecounter medications and natural health products. ${ }^{6}$ An alternative definition, also known as problematic polypharmacy ${ }^{7}$ or medication overload ${ }^{8}$ describes situations in which more medications are used than are indicated or the harms exceed the benefits.

Based on the high prevalence of medication use and polypharmacy, understanding and improving medication management is essential. Medication management 
can be defined as, "patient-centred care to optimize safe, effective and appropriate drug therapy". ${ }^{9}$ Medication management involves a number of services aimed to improve clinical outcomes, such as: completing medication reviews and health assessments, monitoring treatment plans and efficacy and safety of therapy, providing education and promoting self-management. ${ }^{10}$ Self-management is a fundamental component of optimal medication management, and is defined as

an individual's ability to manage the symptoms, treatment, physical and psychosocial consequences and lifestyle changes inherent in living with a chronic condition ( $p$. 178). ${ }^{11}$

Self-management encompasses three key domains: medical, emotional and role management. ${ }^{12}$ Medical management involves tasks and responsibilities related to managing or controlling one's condition (eg taking medication, eating healthy); emotional management involves learning to manage feelings and emotions related to one's condition (eg fear, depression, anger); and role management includes adapting or creating new responsibilities as a result of one's condition (eg scheduling around medication-taking, limiting or finding new hobbies). ${ }^{12}$

Over the past thirty years, the promotion of self-management of chronic conditions through programs and interventions has become increasingly common. ${ }^{13-17}$ Selfmanagement programs and interventions commonly focus on the following areas to improve individuals' clinical and psychosocial outcomes: education, goal setting, decisionmaking, seeking support and resources, self-advocating, problem solving and relationship building. ${ }^{12,18}$ Several systematic and meta reviews have noted positive outcomes of self-management programs and interventions on health outcomes for persons with diabetes, ${ }^{14,16,17}$ heart failure,19,20 asthma $^{21,22}$ and depression. ${ }^{23}$ These systematic and meta reviews included studies that ranged in quality, according to the authors' quality assessments, from low quality to very high quality.

Surprisingly, there has been limited integration of medication management and self-management, despite the effectiveness of self-management interventions and it being a fundamental component of medication management. To date, the majority of medication management frameworks are focused on patient adherence to a specific medication regimen, with a limited focus on broader concepts of self-management. Therefore, given the high use of medications and narrow focus of medication management, this scoping review sought to identify what is reported in the literature on medication management frameworks or models within the context of self-management.

\section{Materials and Methods}

A scoping review was conducted to examine the extent, range and nature of research on medication self-management frameworks, summarize current research and identify gaps in existing literature on this topic. ${ }^{24}$ This scoping review followed the methodological approach described by Levac et al25 and aligns with the methodological guidance of the Joanna Briggs Institute on conducting scoping reviews. ${ }^{26}$ The reporting guidelines of the PRISMA-ScR checklist were also followed (see Supplementary Table 1). ${ }^{27}$ A protocol was developed and registered on Open Science Framework (https://osf.io/6ckg7).

\section{Stage I: Identifying the Research Question}

The research question guiding this scoping review was: what is reported in the literature on patient- or person-level medication management frameworks or models within the context of self-management? The three main objectives were to: (a) examine the extent to which the principles of self-management were integrated into the frameworks, (b) synthesize the key aspects of self-management principles that were captured in medication management frameworks and (c) identify any gaps in the literature to propose future studies.

\section{Stage 2: Identifying Relevant Studies}

Relevant studies were identified by searching four electronic databases (Medline (Ovid Interface), Embase (Ovid Interface), Cumulative Index to Nursing and Allied Health Literature (EBSCO Interface) and Cochrane Library from January 1, 2000, to January 8, 2020. The search strategy was developed in consultation with a librarian at the University of Toronto and adapted for each database. Keywords and medical subject headings, such as medication management, framework, model and self-management, were combined with Boolean and proximity operators (see Supplementary Table 2 for Medline search strategy). The search of electronic databases was conducted on January 8, 2020. In addition to database searches, grey literature was searched on TSpace (University of Toronto research repository) and relevant healthcare and government organization websites (eg 
World Health Organization, National Institute of Health). Reference lists of included articles were reviewed to ensure the inclusion of all relevant literature.

\section{Stage 3: Study Selection}

For inclusion in the scoping review, articles were required to meet the following criteria: (a) described a model or framework originally developed or adapted by authors for medication management; (b) included components of selfmanagement; (c) peer-reviewed literature or grey literature; and (d) published between January 1, 2000, and January 8, 2020. The search was limited to the year 2000 because the majority of research on self-management occurred after this date. ${ }^{12}$ Articles were excluded if any of the following criteria were met: (a) described models of care (interventions, programs, etc.) that were not guided by a concrete model/framework related to medication selfmanagement; (b) models/frameworks that had not been adapted to include components of medication management or self-management; (c) the model/framework was lacking details on what changes/adaptations were made to the model/framework for medication self-management; (d) models/frameworks that only included self-efficacy for medication-taking behaviour as the self-management component; (e) opinion pieces; and (f) conference abstracts or articles in which the full-text was not accessible.

Study selection was conducted using EndNote X8 for de-duplication (reference manager software) and Covidence for screening (online review management software). Following Bramer's method, EndNote X8 was used to de-duplicate the exported articles from the database searches. ${ }^{28}$ The core study team (SJTG, LC, KAJ, TR) used a spreadsheet in Microsoft Excel 2016 to facilitate an interrater screen of a subset of titles and abstracts. All disagreements were discussed until consensus was reached and revisions were made to the eligibility criteria, as needed. Once good agreement (defined as $>0.80)^{29}$ was achieved, the remainder of the titles and abstracts were imported into Covidence and divided among three reviewers (LC, KAJ, TR) to be screened by a single reviewer. After the completion of title and abstract screen, the core study team (SJTG, LC, TR, SRC) completed an interrater screen of 100 full-text articles (in subsets of 25) to test their agreement. Following each subset of 25 fulltext articles, percent agreement was calculated and clarifications were made to the eligibility criteria as needed. After achieving good agreement $(>0.80)$, all full-text articles were double-screened (two separate reviewers screened each article). Disagreements were reviewed by a third screener (senior author) to make the final decision on inclusion.

\section{Stage 4: Charting the Data}

A data extraction sheet was created in Microsoft Excel 2016, which was piloted by the extractors (LC, TR, SRC) to ensure consistency in the data extracted from the articles. Questions and disagreements were discussed until clarification or consensus was achieved. A spot check (review of extracted data) was completed (LC, TR) on $15 \%$ of the articles to ensure the accuracy of extracted information. The following data were extracted from each article: general information, study characteristics, population characteristics, framework characteristics, intervention characteristics, study outcomes and key findings.

\section{Stage 5: Collating, Summarizing and Reporting the Results}

Extracted data were compared across variables. Descriptive numerical analysis and thematic analysis were conducted and are presented below. The data were also critically analyzed to identify current gaps in the literature and develop suggestions for future research, practice and policy.

\section{Results}

\section{Search Results}

The database searches identified 8683 articles and no additional articles were found from the grey literature searches (see Figure 1). Following the removal of duplicates, 5668 articles remained for title and abstract screening. The full-text of 426 articles were reviewed. Following full-text review, 39 articles met all of the criteria and were included in the scoping review (see Table 1 for study characteristics of included articles).

\section{Study Characteristics}

There was a fairly even distribution between quantitative $(\mathrm{n}=15)^{30-44}$ and qualitative $(\mathrm{n}=12)^{45-56}$ study designs, with few mixed method studies $(n=3)^{57-59}$ and several other article types (eg reviews; $n=9) .{ }^{60-68}$ From 2013 to 2019, there was an increase in the number of articles published yearly (total $=24$ ), ${ }^{30-32,36-42,47-51,54,55,57-61,64,67}$ when compared to the 11 years prior (2002 to 2012; total $=15) .{ }^{33-35,43-46,52,53,56,62,63,65,66,68}$ The studies identified were conducted across 12 different countries: the 


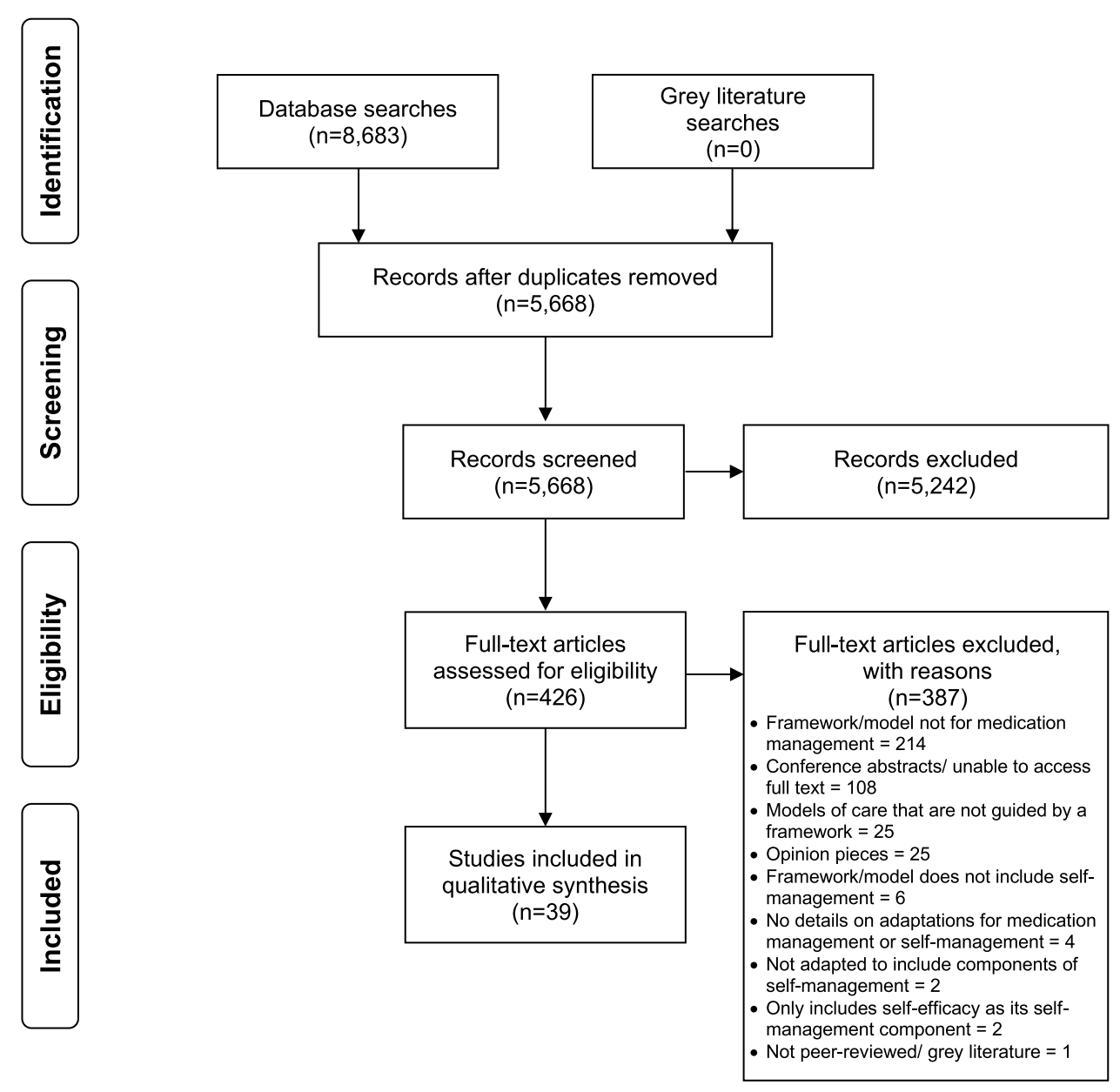

Figure I PRISMA flow diagram of included articles.

United States $(n=24), 30,31,33,34,36,39,40,43-46,48,50,51,53,56,59-$ $63,65,67,68$ China $(n=2),{ }^{38,42}$ the Netherlands $(n=2),{ }^{35,55}$ Singapore $(n=2),{ }^{49,64}$ South Africa $(n=2),{ }^{37,47}$ South Africa and Kenya $(n=1),{ }^{57}$ Australia $(n=1),{ }^{66}$ Canada $(n=1),{ }^{41}$ Israel $(n=1),{ }^{52}$ Malaysia $(n=1),{ }^{58}$ Philippines $(n=1)^{32}$ and the United Kingdom $(n=1) .^{54}$ Of the 39 articles, only one study involved more than one country. $^{57}$

The majority of frameworks or models were developed, adapted or applied to adults and older adults, but two were for a younger population (adolescents and young adults). ${ }^{36,39}$ The most common target populations were adults with HIV $(\mathrm{n}=10)^{30,36,37,43-45,53,57,63}$ and cardiovascular disease $(n=5) .^{46,49-51,65}$ Of the included articles that had a study population $(n=30)$, the majority reported the age and sex or gender of participants. ${ }^{30-38,41-46,51,56}$ There was a fairly even distribution of males and females, with mean ages ranging from 21 to 81 years. Other demographic characteristics reported in about half of the studies

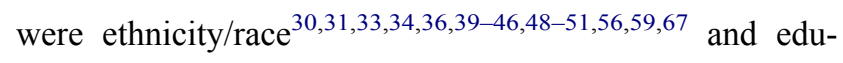
cation level. ${ }^{30-38,41-46,51,55,56}$ The most common ethnicities/races studied were Caucasian and African American among individuals who had at least some college education. Demographic characteristics that were not consistently reported included: income level, marital status, household composition, employment status and comorbidities. The overall sample sizes ranged from 6 to 2213 (mean=265; median=136).

\section{Framework Characteristics}

Approximately half of the included articles developed and presented a new framework $(n=20)$, while the other half adapted previously existing frameworks, or applied them to their findings $(n=19$; see Table 2 for framework characteristics). New frameworks and models were developed by research teams through the use of statistical modeling (structural equation models, confirmatory factor models, measurement models, path analysis; $n=8$ ), data collected 
Table I Characteristics of Included Articles $(n=39)$

\begin{tabular}{|c|c|c|c|c|c|c|}
\hline $\begin{array}{l}\text { Author } \\
\text { (Year) }\end{array}$ & Country & Objective & $\begin{array}{l}\text { Method } \\
\text { Study } \\
\text { Design }\end{array}$ & Participants & $\begin{array}{l}\text { Sample } \\
\text { Size }\end{array}$ & Key Findings \\
\hline $\begin{array}{l}\text { Alsalman and } \\
\text { Smith } \\
(2013)^{60}\end{array}$ & $\begin{array}{l}\text { United } \\
\text { States }\end{array}$ & $\begin{array}{l}\text { - To critique adherence } \\
\text { concepts and terminology } \\
\text { - To present a new medica- } \\
\text { tion-taking behaviour } \\
\text { framework } \\
\text { - To identify future applica- } \\
\text { tions of the new } \\
\text { framework }\end{array}$ & $\begin{array}{l}\text { Neither }{ }^{\dagger} \\
\text { Review }\end{array}$ & NR & $\mathrm{N} / \mathrm{A}$ & $\begin{array}{l}\text { - The framework empha- } \\
\text { sizes a patient-centred } \\
\text { approach and highlights } \\
\text { current challenges with } \\
\text { medication-taking data }\end{array}$ \\
\hline $\begin{array}{l}\text { Archiopoli, } \\
\text { et al }(2016)^{30}\end{array}$ & $\begin{array}{l}\text { United } \\
\text { States }\end{array}$ & $\begin{array}{l}\text { - To analyze the impact of a } \\
\text { model consisting of com- } \\
\text { municative factors, health } \\
\text { behaviour factors and } \\
\text { medication self-efficacy on } \\
\text { medication adherence }\end{array}$ & $\begin{array}{l}\text { Quantitative } \\
\text { Cross- } \\
\text { sectional } \\
\text { study }\end{array}$ & $\begin{array}{l}\text { People living } \\
\text { with HIV }\end{array}$ & 334 & $\begin{array}{l}\text { - Patient-provider communi- } \\
\text { cation is positively asso- } \\
\text { ciated with medication } \\
\text { self-efficacy } \\
\text { - Medication self-efficacy is } \\
\text { positively associated with } \\
\text { adherence }\end{array}$ \\
\hline $\begin{array}{l}\text { Bartlett Ellis } \\
\text { and Welch } \\
(2017)^{61}\end{array}$ & $\begin{array}{l}\text { United } \\
\text { States }\end{array}$ & $\begin{array}{l}\text { - To identify behaviours } \\
\text { associated with medica- } \\
\text { tion-taking and medica- } \\
\text { tion adherence that have } \\
\text { been reported in qualita- } \\
\text { tive studies }\end{array}$ & $\begin{array}{l}\text { Neither } \\
\text { Meta- } \\
\text { ethnographic } \\
\text { qualitative } \\
\text { literature } \\
\text { review }\end{array}$ & $\begin{array}{l}\text { Adults with } \\
\text { chronic kidney } \\
\text { disease and } \\
\text { multiple co- } \\
\text { existing chronic } \\
\text { conditions }\end{array}$ & $\begin{array}{l}\text { I08 (total } \\
\text { across all } \\
\text { included } \\
\text { studies) }\end{array}$ & $\begin{array}{l}\text { - Medication-taking and } \\
\text { medication adherence } \\
\text { behaviours were identified } \\
\text { in } 3 \text { contexts: patient-pro- } \\
\text { vider clinical encounters; } \\
\text { pharmacy encounters; and } \\
\text { day-to-day management }\end{array}$ \\
\hline $\begin{array}{l}\text { Beusterien } \\
\text { et al }(2008)^{45}\end{array}$ & $\begin{array}{l}\text { United } \\
\text { States }\end{array}$ & $\begin{array}{l}\text { - To explore factors and } \\
\text { medication characteristics } \\
\text { that impact medication } \\
\text { adherence }\end{array}$ & $\begin{array}{l}\text { Qualitative } \\
\text { Descriptive }\end{array}$ & $\begin{array}{l}\text { People living } \\
\text { with HIV }\end{array}$ & 35 & $\begin{array}{l}\text { - Six themes related to } \\
\text { medication adherence } \\
\text { were identified: regimen } \\
\text { complexity/medication } \\
\text { features; lifestyle fit; emo- } \\
\text { tional impacts; side effects; } \\
\text { effectiveness; and } \\
\text { communication }\end{array}$ \\
\hline $\begin{array}{l}\text { Blalock } \\
(20 \mathrm{II})^{62}\end{array}$ & $\begin{array}{l}\text { United } \\
\text { States }\end{array}$ & $\begin{array}{l}\text { To explain how beha- } \\
\text { vioural science theories } \\
\text { may enhance the quality of } \\
\text { medication use among } \\
\text { prescription medication } \\
\text { users }\end{array}$ & $\begin{array}{l}\text { Neither } \\
\text { Review }\end{array}$ & NR & $\mathrm{N} / \mathrm{A}$ & $\begin{array}{l}\text { - The quality of research on } \\
\text { medication use could be } \\
\text { improved with an ecologi- } \\
\text { cal model focused on } \\
\text { medication self- } \\
\text { management }\end{array}$ \\
\hline $\begin{array}{l}\text { Bright and } \\
\text { Stanton } \\
(2018)^{31}\end{array}$ & $\begin{array}{l}\text { United } \\
\text { States }\end{array}$ & $\begin{array}{l}\text { - To expand and test a } \\
\text { model of adherence that } \\
\text { involves approach- } \\
\text { oriented coping strategies }\end{array}$ & $\begin{array}{l}\text { Quantitative } \\
\text { Longitudinal }\end{array}$ & $\begin{array}{l}\text { Women with } \\
\text { breast cancer } \\
\text { receiving their } \\
\text { first endocrine } \\
\text { therapy } \\
\text { treatment }\end{array}$ & 130 & $\begin{array}{l}\text { - Support for depressive } \\
\text { symptoms and social fac- } \\
\text { tors are importance con- } \\
\text { siderations for medication } \\
\text { adherence }\end{array}$ \\
\hline
\end{tabular}

(Continued) 
Table I (Continued).

\begin{tabular}{|c|c|c|c|c|c|c|}
\hline $\begin{array}{l}\text { Author } \\
\text { (Year) }\end{array}$ & Country & Objective & $\begin{array}{l}\text { Method } \\
\text { Study } \\
\text { Design }\end{array}$ & Participants & $\begin{array}{l}\text { Sample } \\
\text { Size }\end{array}$ & Key Findings \\
\hline $\begin{array}{l}\text { Brown et al } \\
(2012)^{46}\end{array}$ & $\begin{array}{l}\text { United } \\
\text { States }\end{array}$ & $\begin{array}{l}\text { - To explore factors that } \\
\text { help atrial fibrillation } \\
\text { patients take medications } \\
\text { as prescribed } \\
\text { - To develop a model of the } \\
\text { medication adherence } \\
\text { process }\end{array}$ & $\begin{array}{l}\text { Qualitative } \\
\text { Descriptive }\end{array}$ & $\begin{array}{l}\text { Persons living } \\
\text { with atrial } \\
\text { fibrillation }\end{array}$ & 38 & $\begin{array}{l}\text { - The model integrates the } \\
\text { patient voice and displays } \\
\text { many factors and com- } \\
\text { plexities with medication } \\
\text { adherence }\end{array}$ \\
\hline $\begin{array}{l}\text { Coleman et al } \\
(2014)^{47}\end{array}$ & South Africa & $\begin{array}{l}\text { To explore medication } \\
\text { adherence and propose a } \\
\text { mobile phone medication } \\
\text { adherence monitoring } \\
\text { framework }\end{array}$ & $\begin{array}{l}\text { Qualitative } \\
\text { Case study }\end{array}$ & $\begin{array}{l}\text { Elderly people } \\
\text { living in } \\
\text { retirement } \\
\text { villages }\end{array}$ & 12 & $\begin{array}{l}\text { - A high percent of elderly } \\
\text { people are non-adherent } \\
\text { - The monitoring frame- } \\
\text { work could improve } \\
\text { adherence by sending and } \\
\text { escalating reminders }\end{array}$ \\
\hline $\begin{array}{l}\text { Corneli et al } \\
(2016)^{57}\end{array}$ & $\begin{array}{l}\text { South Africa } \\
\text { and Kenya }\end{array}$ & $\begin{array}{l}\text { To explore reasons for } \\
\text { medication non-adher- } \\
\text { ence and factors impact- } \\
\text { ing decisions not to } \\
\text { adhere }\end{array}$ & $\begin{array}{l}\text { Mixed } \\
\text { Method } \\
\text { Exploratory } \\
\text { cross- } \\
\text { sectional } \\
\text { study }\end{array}$ & $\begin{array}{l}\text { Women who } \\
\text { participated in } \\
\text { the FEM-PrEP } \\
\text { trial }\end{array}$ & $\begin{array}{l}\text { Qualitative: } \\
88 \\
\text { Quantitative: } \\
224\end{array}$ & $\begin{array}{l}\text { - Reasons for medication } \\
\text { non-adherence were } \\
\text { grounded in } 5 \text { areas: indi- } \\
\text { vidual factors; trial charac- } \\
\text { teristics and study pill } \\
\text { regimen; patient-provider } \\
\text { relationship; clinical set- } \\
\text { ting; and the disease }\end{array}$ \\
\hline $\begin{array}{l}\text { de Guzman } \\
\text { et al }(20 \mid 3)^{32}\end{array}$ & Philippines & $\begin{array}{l}\text { - To develop and test and } \\
\text { medication adherence } \\
\text { model relative to numer- } \\
\text { ous factors }\end{array}$ & $\begin{array}{l}\text { Quantitative } \\
\text { Cross- } \\
\text { sectional } \\
\text { study }\end{array}$ & Older adults & 325 & $\begin{array}{l}\text { - Factors related to medica- } \\
\text { tion adherence are con- } \\
\text { nected and solutions } \\
\text { should be multi-faceted }\end{array}$ \\
\hline $\begin{array}{l}\text { Dilorio et al } \\
(2004)^{34}\end{array}$ & $\begin{array}{l}\text { United } \\
\text { States }\end{array}$ & $\begin{array}{l}\text { - To analyze a psychosocial } \\
\text { model of medication self- } \\
\text { management }\end{array}$ & $\begin{array}{l}\text { Quantitative } \\
\text { Repeated } \\
\text { measures }\end{array}$ & $\begin{array}{l}\text { Adults with } \\
\text { epilepsy }\end{array}$ & $320^{\ddagger}$ & $\begin{array}{l}\text { - Medication self-manage- } \\
\text { ment is impacted by psy- } \\
\text { chosocial factors (social } \\
\text { support, stigma) }\end{array}$ \\
\hline $\begin{array}{l}\text { Fransen et al } \\
(2009)^{35}\end{array}$ & Netherlands & $\begin{array}{l}\text { - To examine factors that } \\
\text { determine patients' } \\
\text { adherence to short-term } \\
\text { treatment }\end{array}$ & $\begin{array}{l}\text { Quantitative } \\
\text { Prospective } \\
\text { observational } \\
\text { study }\end{array}$ & $\begin{array}{l}\text { Adults with } \\
\text { dyspepsia using } \\
\text { short-term } \\
\text { proton pump } \\
\text { inhibitor } \\
\text { treatment }\end{array}$ & 347 & $\begin{array}{l}\text { - Medication adherence was } \\
\text { affected by concerns with } \\
\text { medications, limited social } \\
\text { support, lower self-effi- } \\
\text { cacy, increased complaints } \\
\text { and beliefs that complaints } \\
\text { could not be changed }\end{array}$ \\
\hline $\begin{array}{l}\text { Ghose et al } \\
(2019)^{48}\end{array}$ & $\begin{array}{l}\text { United } \\
\text { States }\end{array}$ & $\begin{array}{l}\text { To examine how a transi- } \\
\text { tional housing program } \\
\text { for women recently } \\
\text { released from incarcera- } \\
\text { tion impacts medication } \\
\text { adherence }\end{array}$ & $\begin{array}{l}\text { Qualitative } \\
\text { Grounded } \\
\text { theory }\end{array}$ & $\begin{array}{l}\text { Women with } \\
\text { HIV released } \\
\text { from } \\
\text { incarceration }\end{array}$ & 43 & $\begin{array}{l}\text { - Housing initiated pro- } \\
\text { cesses (empowerment, } \\
\text { cognitive and emotional } \\
\text { re-engagement, health- } \\
\text { seeking behaviours) that } \\
\text { improved adherence }\end{array}$ \\
\hline
\end{tabular}

(Continued) 
Table I (Continued).

\begin{tabular}{|c|c|c|c|c|c|c|}
\hline $\begin{array}{l}\text { Author } \\
\text { (Year) }\end{array}$ & Country & Objective & $\begin{array}{l}\text { Method } \\
\text { Study } \\
\text { Design }\end{array}$ & Participants & $\begin{array}{l}\text { Sample } \\
\text { Size }\end{array}$ & Key Findings \\
\hline $\begin{array}{l}\text { Gifford and } \\
\text { Groessl } \\
(2002)^{63}\end{array}$ & $\begin{array}{l}\text { United } \\
\text { States }\end{array}$ & $\begin{array}{l}\text { To propose a framework } \\
\text { and discuss components } \\
\text { of a program for the self- } \\
\text { management of chronic } \\
\text { HIV disease }\end{array}$ & $\begin{array}{l}\text { Neither } \\
\text { Review }\end{array}$ & $\begin{array}{l}\text { People living } \\
\text { with HIV }\end{array}$ & N/A & $\begin{array}{l}\text { - Key components of a self- } \\
\text { management program } \\
\text { include: goal setting; plan- } \\
\text { ning; communication; } \\
\text { information seeking; and } \\
\text { symptom management }\end{array}$ \\
\hline $\begin{array}{l}\text { Goh et al } \\
(2017)^{64}\end{array}$ & Singapore & $\begin{array}{l}\text { - To identify factors affect- } \\
\text { ing adherence and evalu- } \\
\text { ate the association of the } \\
\text { factors with adherence }\end{array}$ & $\begin{array}{l}\text { Neither } \\
\text { Systematic } \\
\text { review }\end{array}$ & $\begin{array}{l}\text { Rheumatic } \\
\text { patients }\end{array}$ & $N / A$ & $\begin{array}{l}\text { - Five main categories } \\
\text { related to adherence } \\
\text { were identified: patient } \\
\text { factors; therapy factors; } \\
\text { condition factors; health } \\
\text { system factors; and social/ } \\
\text { economic factors }\end{array}$ \\
\hline $\begin{array}{l}\text { Guy et al } \\
(2018)^{36}\end{array}$ & $\begin{array}{l}\text { United } \\
\text { States }\end{array}$ & $\begin{array}{l}\text { - To identify the association } \\
\text { between adherence, } \\
\text { emotional support seek- } \\
\text { ing and active coping }\end{array}$ & $\begin{array}{l}\text { Quantitative } \\
\text { Cross- } \\
\text { sectional } \\
\text { study }\end{array}$ & $\begin{array}{l}\text { Young African } \\
\text { American men } \\
\text { who have sex } \\
\text { with men who } \\
\text { have HIV }\end{array}$ & 49 & $\begin{array}{l}\text { - Emotional support seeking } \\
\text { was significantly associated } \\
\text { with adherence through } \\
\text { active coping }\end{array}$ \\
\hline $\begin{array}{l}\text { Koh et al } \\
(2018)^{49}\end{array}$ & Singapore & $\begin{array}{l}\text { - To explore factors affect- } \\
\text { ing medication adherence }\end{array}$ & $\begin{array}{l}\text { Qualitative } \\
\text { Descriptive }\end{array}$ & $\begin{array}{l}\text { Persons living } \\
\text { with } \\
\text { atherosclerotic } \\
\text { cardiovascular } \\
\text { disease }\end{array}$ & 20 & $\begin{array}{l}\text { - Factors affecting medica- } \\
\text { tion adherence included: } \\
\text { complex regimens; lack of } \\
\text { support; perceived ser- } \\
\text { iousness of condition; and } \\
\text { cost }\end{array}$ \\
\hline $\begin{array}{l}\text { Lau et al } \\
(2017)^{38}\end{array}$ & China & $\begin{array}{l}\text { - To examine factors } \\
\text { impacting medication } \\
\text { adherence using structural } \\
\text { equation modeling }\end{array}$ & $\begin{array}{l}\text { Quantitative } \\
\text { Exploratory } \\
\text { cross- } \\
\text { sectional } \\
\text { study }\end{array}$ & $\begin{array}{l}\text { Older Chinese } \\
\text { community- } \\
\text { dwelling adults }\end{array}$ & 593 & $\begin{array}{l}\text { - Improved medication } \\
\text { adherence was associated } \\
\text { with fewer chronic dis- } \\
\text { eases; more medication } \\
\text { categories; lower fear of } \\
\text { sharing scores; and higher } \\
\text { willingness to share and } \\
\text { self-care agency scores }\end{array}$ \\
\hline $\begin{array}{l}\text { Li et al } \\
(2007)^{65}\end{array}$ & $\begin{array}{l}\text { United } \\
\text { States }\end{array}$ & $\begin{array}{l}\text { - To describe a theoretical } \\
\text { framework for antihyper- } \\
\text { tensive medication } \\
\text { compliance }\end{array}$ & $\begin{array}{l}\text { Neither } \\
\text { Review }\end{array}$ & $\begin{array}{l}\text { Chinese } \\
\text { immigrants } \\
\text { with } \\
\text { hypertension }\end{array}$ & $N / A$ & $\begin{array}{l}\text { - The framework was built } \\
\text { on four constructs: moti- } \\
\text { vation; cultural health per- } \\
\text { ceptions; modifying } \\
\text { factors; and cultural health } \\
\text { activities }\end{array}$ \\
\hline $\begin{array}{l}\text { McCann et al } \\
(2008)^{66}\end{array}$ & Australia & $\begin{array}{l}\text { - To present a model for } \\
\text { medication adherence } \\
\text { self-efficacy for chronic } \\
\text { mental illness }\end{array}$ & $\begin{array}{l}\text { Neither } \\
\text { Review }\end{array}$ & $\begin{array}{l}\text { Individuals with } \\
\text { chronic mental } \\
\text { illness }\end{array}$ & $N / A$ & $\begin{array}{l}\text { - Medication adherence is } \\
\text { multidimensional and } \\
\text { includes factors related to } \\
\text { self-efficacy, relationships, } \\
\text { and social, psychological } \\
\text { and environmental support }\end{array}$ \\
\hline
\end{tabular}

(Continued) 
Table I (Continued).

\begin{tabular}{|c|c|c|c|c|c|c|}
\hline $\begin{array}{l}\text { Author } \\
\text { (Year) }\end{array}$ & Country & Objective & $\begin{array}{l}\text { Method } \\
\text { Study } \\
\text { Design }\end{array}$ & Participants & $\begin{array}{l}\text { Sample } \\
\text { Size }\end{array}$ & Key Findings \\
\hline $\begin{array}{l}\text { Mickelson } \\
\text { et al }(2016)^{50}\end{array}$ & $\begin{array}{l}\text { United } \\
\text { States }\end{array}$ & $\begin{array}{l}\text { - To describe and analyze } \\
\text { the medication manage- } \\
\text { ment of older adults with } \\
\text { heart failure using the } \\
\text { macrocognitive workflow } \\
\text { framework }\end{array}$ & $\begin{array}{l}\text { Qualitative } \\
\text { Descriptive }\end{array}$ & $\begin{array}{l}\text { Older adults } \\
\text { with heart } \\
\text { failure }\end{array}$ & 61 & $\begin{array}{l}\text { - Macrocognitive processes } \\
\text { affected medication man- } \\
\text { agement and were often } \\
\text { collaborative }\end{array}$ \\
\hline $\begin{array}{l}\text { Nantha et al } \\
(2019)^{58}\end{array}$ & Malaysia & $\begin{array}{l}\text { - To create an integrated } \\
\text { behavioural model for } \\
\text { diabetic medication } \\
\text { compliance }\end{array}$ & $\begin{array}{l}\text { Mixed } \\
\text { Method } \\
\text { Protocol } \\
\text { Cross- } \\
\text { sectional } \\
\text { study }\end{array}$ & $\begin{array}{l}\text { Adults with } \\
\text { type } 2 \text { diabetes }\end{array}$ & $\begin{array}{l}\text { Study I: } \\
\text { Expecting } 20 \\
\text { interviews, } \\
10 \text { for focus } \\
\text { groups } \\
\text { Study 2: } \\
\text { Expecting } \\
200\end{array}$ & - N/A \\
\hline $\begin{array}{l}\text { Newman- } \\
\text { Casey et al } \\
(2013)^{67}\end{array}$ & $\begin{array}{l}\text { United } \\
\text { States }\end{array}$ & $\begin{array}{l}\text { - To review educational } \\
\text { interventions, identify } \\
\text { their effect on glaucoma } \\
\text { medication adherence and } \\
\text { how they use Health } \\
\text { Behaviour Theory }\end{array}$ & $\begin{array}{l}\text { Neither } \\
\text { Systematic } \\
\text { Review }\end{array}$ & Glaucoma & N/A & $\begin{array}{l}\text { - There are limited high } \\
\text { quality studies using edu- } \\
\text { cation for glaucoma medi- } \\
\text { cation adherence }\end{array}$ \\
\hline $\begin{array}{l}\text { Paterson et al } \\
(2018)^{41}\end{array}$ & Canada & $\begin{array}{l}\text { To examine the relation- } \\
\text { ship between medication } \\
\text { adherence and depressive } \\
\text { symptoms, self-efficacy, } \\
\text { problem solving and neu- } \\
\text { rocognitive abilities }\end{array}$ & $\begin{array}{l}\text { Quantitative } \\
\text { Cross- } \\
\text { sectional } \\
\text { study }\end{array}$ & $\begin{array}{l}\text { Renal } \\
\text { transplant } \\
\text { recipients }\end{array}$ & 211 & $\begin{array}{l}\text { - Problem solving and self- } \\
\text { efficacy were positively } \\
\text { associated with medication } \\
\text { adherence }\end{array}$ \\
\hline $\begin{array}{l}\text { Piette et al } \\
(2006)^{68}\end{array}$ & $\begin{array}{l}\text { United } \\
\text { States }\end{array}$ & $\begin{array}{l}\text { - To present a conceptual } \\
\text { framework to understand } \\
\text { influencing factors on } \\
\text { responses to medication } \\
\text { costs }\end{array}$ & $\begin{array}{l}\text { Neither } \\
\text { Review }\end{array}$ & $\begin{array}{l}\text { Chronically ill } \\
\text { patients }\end{array}$ & $N / A$ & $\begin{array}{l}\text { - Medication costs are } \\
\text { impacted by patient and } \\
\text { clinician characteristics, } \\
\text { type of medication, and } \\
\text { the health system }\end{array}$ \\
\hline $\begin{array}{l}\text { Roe et al } \\
(2009)^{52}\end{array}$ & Israel & $\begin{array}{l}\text { - To explore reasons peo- } \\
\text { ple with serious mental } \\
\text { illness stop taking pre- } \\
\text { scription medication }\end{array}$ & $\begin{array}{l}\text { Qualitative } \\
\text { Descriptive }\end{array}$ & $\begin{array}{l}\text { Serious mental } \\
\text { illness }\end{array}$ & 7 & $\begin{array}{l}\text { - Medication non-adherence } \\
\text { is contextual and based on } \\
\text { subjective experiences }\end{array}$ \\
\hline $\begin{array}{l}\text { Schafheutle } \\
\text { et al }(2018)^{54}\end{array}$ & $\begin{array}{l}\text { United } \\
\text { Kingdom }\end{array}$ & $\begin{array}{l}\text { - To describe strategies for } \\
\text { medication-taking follow- } \\
\text { ing hospital discharge } \\
\text { - To identify members of } \\
\text { one's social network } \\
\text { involved in medication- } \\
\text { taking strategies }\end{array}$ & $\begin{array}{l}\text { Qualitative } \\
\text { Descriptive }\end{array}$ & $\begin{array}{l}\text { Chronic } \\
\text { obstructive } \\
\text { pulmonary } \\
\text { disease }\end{array}$ & 12 & $\begin{array}{l}\text { - Strategies for medication- } \\
\text { taking did not change } \\
\text { from pre-hospitalization } \\
\text { - Social networks assisting } \\
\text { with medication-taking } \\
\text { were: family and health- } \\
\text { care professionals }\end{array}$ \\
\hline
\end{tabular}

(Continued) 
Table I (Continued).

\begin{tabular}{|c|c|c|c|c|c|c|}
\hline $\begin{array}{l}\text { Author } \\
\text { (Year) }\end{array}$ & Country & Objective & $\begin{array}{l}\text { Method } \\
\text { Study } \\
\text { Design }\end{array}$ & Participants & $\begin{array}{l}\text { Sample } \\
\text { Size }\end{array}$ & Key Findings \\
\hline $\begin{array}{l}\text { Schrijvers } \\
\text { et al }(20 \text { I4 })^{55}\end{array}$ & Netherlands & $\begin{array}{l}\text { - To describe the adher- } \\
\text { ence process from the } \\
\text { patients' perspective }\end{array}$ & $\begin{array}{l}\text { Qualitative } \\
\text { Grounded } \\
\text { Theory }\end{array}$ & Hemophilia & 21 & $\begin{array}{l}\text { Adherence was impacted } \\
\text { by: acceptance of hemo- } \\
\text { philia; feelings and fearing } \\
\text { symptoms; understanding } \\
\text { of hemophilia and prophy- } \\
\text { laxis; and behavior }\end{array}$ \\
\hline $\begin{array}{l}\text { Simoni et al } \\
(2006)^{43}\end{array}$ & $\begin{array}{l}\text { United } \\
\text { States }\end{array}$ & $\begin{array}{l}\text { - To test a model of adher- } \\
\text { ence among inner-city } \\
\text { HIV-positive patients on } \\
\text { antiretroviral therapy }\end{array}$ & $\begin{array}{l}\text { Quantitative } \\
\text { Longitudinal } \\
\text { Evaluation }\end{array}$ & $\begin{array}{l}\text { HIV-Positive } \\
\text { Men and } \\
\text { Women on } \\
\text { Antiretroviral } \\
\text { Therapy }\end{array}$ & 136 & $\begin{array}{l}\text { - Baseline adherence self- } \\
\text { efficacy predicted patients' } \\
\text { self-reported adherence }\end{array}$ \\
\hline $\begin{array}{l}\text { Swanlund } \\
\text { et al }(2008)^{56}\end{array}$ & $\begin{array}{l}\text { United } \\
\text { States }\end{array}$ & $\begin{array}{l}\text { - To explore medication } \\
\text { self-management among } \\
\text { older adults }\end{array}$ & $\begin{array}{l}\text { Qualitative } \\
\text { Descriptive }\end{array}$ & $\begin{array}{l}\text { Community- } \\
\text { dwelling older } \\
\text { adults }\end{array}$ & 19 & $\begin{array}{l}\text { - Self-management capability } \\
\text { is positively impacted by: } \\
\text { living orderly and aging } \\
\text { well }\end{array}$ \\
\hline $\begin{array}{l}\text { Weaver et al } \\
(2005)^{44}\end{array}$ & $\begin{array}{l}\text { United } \\
\text { States }\end{array}$ & $\begin{array}{l}\text { To study and test a stress } \\
\text { and coding model of fac- } \\
\text { tors associated with } \\
\text { adherence and HIV viral } \\
\text { load }\end{array}$ & $\begin{array}{l}\text { Quantitative } \\
\text { Secondary } \\
\text { analysis of a } \\
\text { longitudinal } \\
\text { randomized } \\
\text { trial }\end{array}$ & $\begin{array}{l}\text { Adults with } \\
\text { HIV }\end{array}$ & 322 & $\begin{array}{l}\text { - Adherence is predicted by } \\
\text { avoidance-oriented coping, } \\
\text { which is impacted by social } \\
\text { support and negative } \\
\text { mood }\end{array}$ \\
\hline $\begin{array}{l}\text { Saslo } \\
(2007)^{53}\end{array}$ & $\begin{array}{l}\text { United } \\
\text { States }\end{array}$ & $\begin{array}{l}\text { - To observe the culture of } \\
\text { living with HIV and its } \\
\text { impact on adherence to } \\
\text { care plans }\end{array}$ & $\begin{array}{l}\text { Qualitative } \\
\text { Ethnography }\end{array}$ & $\begin{array}{l}\text { Adults with } \\
\text { HIV }\end{array}$ & 6 & $\begin{array}{l}\text { - Adherence was impacted } \\
\text { by: literacy; fear; disease; } \\
\text { and policies }\end{array}$ \\
\hline $\begin{array}{l}\text { Qu et al } \\
(20 \mid 8)^{42}\end{array}$ & China & $\begin{array}{l}\text { - To examine adherence to } \\
\text { pre-exposure prophylaxis } \\
\text { - To identify if information, } \\
\text { motivation and behavioural } \\
\text { skills are significantly asso- } \\
\text { ciated with adherence } \\
\text { - To explore objective fac- } \\
\text { tors for non-adherence } \\
\text { to pre-exposure } \\
\text { prophylaxis } \\
\text { - To describe factors of } \\
\text { non-adherence for future } \\
\text { interventions }\end{array}$ & $\begin{array}{l}\text { Quantitative } \\
\text { Secondary } \\
\text { analysis of } \\
\text { randomized } \\
\text { placebo- } \\
\text { controlled } \\
\text { trial }\end{array}$ & $\begin{array}{l}\text { Men who have } \\
\text { sex with men } \\
\text { (at risk of } \\
\text { contracting } \\
\text { HIV) }\end{array}$ & 331 & $\begin{array}{l}\text { - Adherence was not asso- } \\
\text { ciated with social psycho- } \\
\text { logical factors in the model }\end{array}$ \\
\hline $\begin{array}{l}\text { O'Brien } \\
(2014)^{59}\end{array}$ & $\begin{array}{l}\text { United } \\
\text { States }\end{array}$ & $\begin{array}{l}\text { - To identify factors asso- } \\
\text { ciated with medication } \\
\text { adherence and non- } \\
\text { adherence } \\
\text { - To describe medication } \\
\text { self-management } \\
\text { processes }\end{array}$ & $\begin{array}{l}\text { Mixed } \\
\text { Method } \\
\text { Descriptive } \\
\text { explanatory } \\
\text { study }\end{array}$ & Older adults & 268 & $\begin{array}{l}\text { - Assistance from caregivers } \\
\text { positively impacted medi- } \\
\text { cation adherence }\end{array}$ \\
\hline
\end{tabular}

(Continued) 
Table I (Continued).

\begin{tabular}{|c|c|c|c|c|c|c|}
\hline $\begin{array}{l}\text { Author } \\
\text { (Year) }\end{array}$ & Country & Objective & $\begin{array}{l}\text { Method } \\
\text { Study } \\
\text { Design }\end{array}$ & Participants & $\begin{array}{l}\text { Sample } \\
\text { Size }\end{array}$ & Key Findings \\
\hline $\begin{array}{l}\text { Mondesir } \\
\text { et al }(2019)^{51}\end{array}$ & $\begin{array}{l}\text { United } \\
\text { States }\end{array}$ & $\begin{array}{l}\text { To explore influences on } \\
\text { medication adherence } \\
\text { using the World Health } \\
\text { Organization } \\
\text { Multidimensional } \\
\text { Adherence Model }\end{array}$ & $\begin{array}{l}\text { Qualitative } \\
\text { Descriptive }\end{array}$ & $\begin{array}{l}\text { Adults with } \\
\text { coronary heart } \\
\text { disease }\end{array}$ & 18 & $\begin{array}{l}\text { - Adherence was impacted } \\
\text { by factors related to the: } \\
\text { patient; social and eco- } \\
\text { nomic; therapy; and health } \\
\text { system }\end{array}$ \\
\hline $\begin{array}{l}\text { Messina et al } \\
(2016)^{40}\end{array}$ & $\begin{array}{l}\text { United } \\
\text { States }\end{array}$ & $\begin{array}{l}\text { - To examine motivational } \\
\text { constructs for non-medi- } \\
\text { cal use of pain relievers, } \\
\text { stimulants and sedatives/ } \\
\text { tranquilizers }\end{array}$ & $\begin{array}{l}\text { Quantitative } \\
\text { Cross- } \\
\text { sectional } \\
\text { study }\end{array}$ & $\begin{array}{l}\text { Undergraduate } \\
\text { students }\end{array}$ & 1016 & $\begin{array}{l}\text { - Non-medical use of pain } \\
\text { relievers, stimulants and } \\
\text { sedatives/tranquilizers may } \\
\text { be driven by self-treatment } \\
\text { and recreational use }\end{array}$ \\
\hline $\begin{array}{l}\text { MacDonell } \\
\text { et al }(2016)^{39}\end{array}$ & $\begin{array}{l}\text { United } \\
\text { States }\end{array}$ & $\begin{array}{l}\text { - To test a model of medi- } \\
\text { cation adherence, specific } \\
\text { to social cognitive factors }\end{array}$ & $\begin{array}{l}\text { Quantitative } \\
\text { Cross- } \\
\text { sectional } \\
\text { study }\end{array}$ & $\begin{array}{l}\text { Ethnic and } \\
\text { racial minority } \\
\text { HIV-positive } \\
\text { youth }\end{array}$ & 2213 & $\begin{array}{l}\text { - Adherence was positively } \\
\text { associated with higher } \\
\text { self-efficacy and readiness } \\
\text { - Adherence was negatively } \\
\text { associated with substance } \\
\text { use and psychological } \\
\text { symptoms }\end{array}$ \\
\hline $\begin{array}{l}\text { Kekwaletswe } \\
\text { et al }(2017)^{37}\end{array}$ & South Africa & $\begin{array}{l}\text { - To duplicate and build on } \\
\text { a multicomponent model } \\
\text { of antiretroviral therapy } \\
\text { adherence }\end{array}$ & $\begin{array}{l}\text { Quantitative } \\
\text { Cross- } \\
\text { sectional } \\
\text { study }\end{array}$ & $\begin{array}{l}\text { HIV positive } \\
\text { people taking } \\
\text { antiretroviral } \\
\text { therapy }\end{array}$ & 304 & $\begin{array}{l}\text { - Adherence was associated } \\
\text { with: reduced depression; } \\
\text { reduced alcohol use; and } \\
\text { higher self-efficacy beliefs }\end{array}$ \\
\hline $\begin{array}{l}\text { Dehn } \\
(2010)^{33}\end{array}$ & $\begin{array}{l}\text { United } \\
\text { States }\end{array}$ & $\begin{array}{l}\text { - To clarify the association } \\
\text { of adherence to cystic } \\
\text { fibrosis treatments to } \\
\text { numerous factors }\end{array}$ & $\begin{array}{l}\text { Quantitative } \\
\text { Descriptive } \\
\text { and } \\
\text { correlational }\end{array}$ & $\begin{array}{l}\text { Adults with } \\
\text { cystic fibrosis }\end{array}$ & 173 & $\begin{array}{l}\text { - Adherence was signifi- } \\
\text { cantly associated with: } \\
\text { problem-focused coping } \\
\text { and physician partnership }\end{array}$ \\
\hline
\end{tabular}

Notes: ${ }^{\dagger}$ The article was not original research (ie, qualitative, quantitative or mixed methods). ${ }^{\ddagger}$ Three participants were excluded from all analyses (incomplete assessments or withdrawal post-baseline).

Abbreviations: NR, not reported; N/A, not applicable; HIV, human immunodeficiency virus.

from interviews and focus groups $(\mathrm{n}=6)$ and literature syntheses $(n=4)$. One new framework was developed from literature syntheses and focus groups and one framework lacked sufficient details on how it was developed. Adapted frameworks were most commonly based on social cognitive theory, behaviour change models and medication adherence models. Similar to newly developed frameworks, adapted frameworks were often based on quantitative surveys $(n=7)$, literature syntheses $(n=4)$, qualitative data from interviews and focus groups $(n=3)$ or a combination $(n=3)$. No frameworks were developed or adapted through co-design sessions with participants.
The majority of self-management domains (eg adherence; self-efficacy; depression; and seeking advice, support and relationships) included in the frameworks were categorized as medical management (eg medication/treatment adherence, medication/adherence self-efficacy, medication-taking behaviour), followed by emotional management (eg depression, emotional impact) and role management (eg problem solving, day-to-day management, spirituality). Across all frameworks, developed, adapted and applied, the most common domains (not limited to self-management) were related to adherence, selfefficacy, depression, social support and regimen 
Table 2 Framework Characteristics

\begin{tabular}{|c|c|c|c|c|}
\hline $\begin{array}{l}\text { Author } \\
\text { (Year) }\end{array}$ & $\begin{array}{l}\text { Name of Framework } / \\
\text { Model }\end{array}$ & Description & Target Population & $\begin{array}{l}\text { New or } \\
\text { Adapted } \\
\text { Framework }\end{array}$ \\
\hline $\begin{array}{l}\text { Alsalman and } \\
\text { Smith } \\
(2013)^{60}\end{array}$ & $\begin{array}{l}\text { Medication-Taking } \\
\text { Behavior }\end{array}$ & $\begin{array}{l}\text { - A framework describing all medication- } \\
\text { taking behaviours (how, when and how } \\
\text { much patients take prescribed medica- } \\
\text { tions) using new concepts and measures }\end{array}$ & NR & New \\
\hline $\begin{array}{l}\text { Archiopoli, } \\
\text { et al }(2016)^{30}\end{array}$ & Mediating Model & $\begin{array}{l}\text { - A model examining the relationship } \\
\text { between behavioural health factors and } \\
\text { interpersonal communication factors } \\
\text { with medication self-efficacy and medi- } \\
\text { cation adherence }\end{array}$ & People living with HIV & New \\
\hline $\begin{array}{l}\text { Bartlett Ellis } \\
\text { and Welch } \\
(2017)^{61}\end{array}$ & $\begin{array}{l}\text { Medication-taking across } \\
\text { the care continuum and } \\
\text { adherence-related } \\
\text { outcomes }\end{array}$ & $\begin{array}{l}\text { - A framework to organize medication- } \\
\text { taking behaviours within clinical } \\
\text { encounters, pharmacy encounters and } \\
\text { day-to-day management }\end{array}$ & $\begin{array}{l}\text { Adults with chronic kidney } \\
\text { disease and multiple coexisting } \\
\text { chronic conditions }\end{array}$ & New \\
\hline $\begin{array}{l}\text { Beusterien } \\
\text { et al }(2008)^{45}\end{array}$ & $\begin{array}{l}\text { Patient reported } \\
\text { adherence diagram }\end{array}$ & $\begin{array}{l}\text { - A framework illustrating the relation- } \\
\text { ship of themes (emotional impact, } \\
\text { communication, effectiveness, side } \\
\text { effects, lifestyle fit and regimen com- } \\
\text { plexity/medication features) that have } \\
\text { an influence on adherence to therapy }\end{array}$ & People living with HIV & New \\
\hline $\begin{array}{l}\text { Blalock } \\
(2011)^{62}\end{array}$ & $\begin{array}{l}\text { Proposed ecological } \\
\text { framework for medication } \\
\text { self-management }\end{array}$ & $\begin{array}{l}\text { - A framework based on an ecological } \\
\text { model identifying goals at } 5 \text { levels } \\
\text { (patient, family, providers, system and } \\
\text { social policy/government regulations) } \\
\text { that impact medication use and self- } \\
\text { management }\end{array}$ & NR & Adapted \\
\hline $\begin{array}{l}\text { Bright and } \\
\text { Stanton } \\
(2018)^{31}\end{array}$ & $\begin{array}{l}\text { Stress and coping model of } \\
\text { endocrine therapy } \\
\text { adherence }\end{array}$ & $\begin{array}{l}\text { - A model that incorporates social sup- } \\
\text { port, coping and depressive symptoms } \\
\text { to test their influence on adherence }\end{array}$ & $\begin{array}{l}\text { Women with breast cancer } \\
\text { receiving their first endocrine } \\
\text { therapy treatment }\end{array}$ & New \\
\hline $\begin{array}{l}\text { Brown et al } \\
(2012)^{46}\end{array}$ & $\begin{array}{l}\text { Conceptual model of } \\
\text { adherence to oral } \\
\text { anticoagulants }\end{array}$ & $\begin{array}{l}\text { - A model outlining the adherence pro- } \\
\text { cess to oral anticoagulants (includes } \\
\text { contextual factors, knowledge, motiva- } \\
\text { tion, habit formation, self-efficacy and } \\
\text { adherence) }\end{array}$ & $\begin{array}{l}\text { Persons living with atrial } \\
\text { fibrillation }\end{array}$ & New \\
\hline $\begin{array}{l}\text { Coleman et al } \\
(2014)^{47}\end{array}$ & $\begin{array}{l}\text { Mobile phone adherence } \\
\text { monitoring framework } \\
\text { (MPAMF) }\end{array}$ & $\begin{array}{l}\text { - A mobile health framework for medica- } \\
\text { tion self-efficacy among elderly indivi- } \\
\text { duals, with an adherence monitoring } \\
\text { system }\end{array}$ & $\begin{array}{l}\text { Elderly people living in } \\
\text { retirement villages }\end{array}$ & New \\
\hline $\begin{array}{l}\text { Corneli et al } \\
(2016)^{57}\end{array}$ & $\begin{array}{l}\text { Conceptual model } \\
\text { illustrating factors related } \\
\text { to non-adherence in FEM- } \\
\text { PrEP }\end{array}$ & $\begin{array}{l}\text { - A model of commonly reported factors } \\
\text { related to non-adherence }\end{array}$ & $\begin{array}{l}\text { Women who participated in the } \\
\text { Preexposure Prophylaxis Trial } \\
\text { for HIV Prevention among } \\
\text { African Women (FEM-PrEP) }\end{array}$ & Adapted \\
\hline
\end{tabular}

(Continued) 
Table 2 (Continued).

\begin{tabular}{|c|c|c|c|c|}
\hline $\begin{array}{l}\text { Author } \\
\text { (Year) }\end{array}$ & $\begin{array}{l}\text { Name of Framework } / \\
\text { Model }\end{array}$ & Description & Target Population & $\begin{array}{l}\text { New or } \\
\text { Adapted } \\
\text { Framework }\end{array}$ \\
\hline $\begin{array}{l}\text { de Guzman } \\
\text { et al }(2013)^{32}\end{array}$ & $\begin{array}{l}\text { Emerging model of } \\
\text { medication adherence }\end{array}$ & $\begin{array}{l}\text { - A structural equation model identifying } \\
\text { the relationships that have a positive } \\
\text { effect on medication adherence (beha- } \\
\text { vioural attitude, perceived behavioral } \\
\text { control, subjective norm) }\end{array}$ & Older adults & New \\
\hline $\begin{array}{l}\text { Dilorio et al } \\
(2004)^{34}\end{array}$ & $\begin{array}{l}\text { Modified model of epilepsy } \\
\text { self-management for } \\
\text { medications }\end{array}$ & $\begin{array}{l}\text { - A model of epilepsy self-management } \\
\text { for medications that includes personal, } \\
\text { social and provider-specific variables }\end{array}$ & Adults with epilepsy & Adapted \\
\hline $\begin{array}{l}\text { Fransen et al } \\
(2009)^{35}\end{array}$ & $\begin{array}{l}\text { Theoretical model: } \\
\text { determinants of patient } \\
\text { adherence }\end{array}$ & $\begin{array}{l}\text { - A theoretical model of patient-related } \\
\text { factors impacting adherence to short- } \\
\text { term proton pump inhibitor treatment }\end{array}$ & $\begin{array}{l}\text { Adults with dyspepsia using } \\
\text { short-term proton pump } \\
\text { inhibitor treatment }\end{array}$ & Adapted \\
\hline $\begin{array}{l}\text { Ghose et al } \\
(2019)^{48}\end{array}$ & $\begin{array}{l}\text { Housing's effect on } \\
\text { adherence }\end{array}$ & $\begin{array}{l}\text { - A conceptual framework that includes } \\
\text { subjective and material processes and } \\
\text { examines how housing affects advances } \\
\text { in antiretroviral adherence }\end{array}$ & $\begin{array}{l}\text { Women with HIV released from } \\
\text { incarceration }\end{array}$ & Adapted \\
\hline $\begin{array}{l}\text { Gifford and } \\
\text { Groessl } \\
(2002)^{63}\end{array}$ & $\begin{array}{l}\text { HIV disease self- } \\
\text { management }\end{array}$ & $\begin{array}{l}\text { - A conceptual framework for under- } \\
\text { standing and categorizing determinants } \\
\text { of HIV disease self-management and } \\
\text { health outcomes }\end{array}$ & People living with HIV & Adapted \\
\hline $\begin{array}{l}\text { Goh et al } \\
(2017)^{64}\end{array}$ & $\begin{array}{l}\text { Hand model of medication } \\
\text { adherence in rheumatic } \\
\text { factors }\end{array}$ & $\begin{array}{l}\text { - A model of five categories of factors } \\
\text { that affect medication adherence }\end{array}$ & Rheumatic patients & Adapted \\
\hline $\begin{array}{l}\text { Guy et al } \\
(2018)^{36}\end{array}$ & Mediation model & $\begin{array}{l}\text { - A model examining the association } \\
\text { between active coping, emotional sup- } \\
\text { port seeking and treatment adherence }\end{array}$ & $\begin{array}{l}\text { Young African American men } \\
\text { who have sex with men who } \\
\text { have HIV }\end{array}$ & Adapted \\
\hline $\begin{array}{l}\text { Koh et al } \\
(2018)^{49}\end{array}$ & $\begin{array}{l}\text { Integration of WHO } \\
\text { framework of adherence } \\
\text { and Levesque's framework } \\
\text { for health care access }\end{array}$ & $\begin{array}{l}\text { - A framework for understanding how } \\
\text { system, social, economic and patient } \\
\text { related factors impact medication } \\
\text { adherence }\end{array}$ & $\begin{array}{l}\text { Persons living with } \\
\text { atherosclerotic cardiovascular } \\
\text { disease }\end{array}$ & Adapted \\
\hline $\begin{array}{l}\text { Lau et al } \\
(2017)^{38}\end{array}$ & $\begin{array}{l}\text { Structural equation model } \\
\text { of five-dimensional factors } \\
\text { on medication adherence } \\
\text { among older adults }\end{array}$ & $\begin{array}{l}\text { - A hypothetical model of medication } \\
\text { adherence that incorporates healthcare } \\
\text { team, patient, disease, therapy and } \\
\text { socioeconomic related factors }\end{array}$ & $\begin{array}{l}\text { Older Chinese community- } \\
\text { dwelling adults }\end{array}$ & Adapted \\
\hline $\begin{array}{l}\text { Li et al } \\
(2007)^{65}\end{array}$ & $\begin{array}{l}\text { Modified sick role } \\
\text { behaviour model }\end{array}$ & $\begin{array}{l}\text { - A model consisting of four constructs } \\
\text { to predict their impact on medication } \\
\text { compliance }\end{array}$ & $\begin{array}{l}\text { Chinese immigrants with } \\
\text { hypertension }\end{array}$ & Adapted \\
\hline $\begin{array}{l}\text { McCann et al } \\
(2008)^{66}\end{array}$ & $\begin{array}{l}\text { Self-efficacy model of } \\
\text { medication adherence }\end{array}$ & $\begin{array}{l}\text { - A model of medication adherence that } \\
\text { consists of five core factors and con- } \\
\text { textual influences }\end{array}$ & $\begin{array}{l}\text { Individuals with chronic mental } \\
\text { illness }\end{array}$ & New \\
\hline $\begin{array}{l}\text { Mickelson } \\
\text { et al }(2016)^{50}\end{array}$ & $\begin{array}{l}\text { Macrocognitive workflow } \\
\text { of medication management }\end{array}$ & $\begin{array}{l}\text { - A model outlining the macrocognitive } \\
\text { workflow of medication management } \\
\text { and includes artifacts, actors and actions }\end{array}$ & Older adults with heart failure & Adapted \\
\hline
\end{tabular}

(Continued) 
Table 2 (Continued).

\begin{tabular}{|c|c|c|c|c|}
\hline $\begin{array}{l}\text { Author } \\
\text { (Year) }\end{array}$ & $\begin{array}{l}\text { Name of Framework } / \\
\text { Model }\end{array}$ & Description & Target Population & $\begin{array}{l}\text { New or } \\
\text { Adapted } \\
\text { Framework }\end{array}$ \\
\hline $\begin{array}{l}\text { Nantha et al } \\
(2019)^{58}\end{array}$ & $\begin{array}{l}\text { Integrated behavioural } \\
\text { model of medication } \\
\text { compliance }\end{array}$ & $\begin{array}{l}\text { - An integrated behavioural model of } \\
\text { medication compliance that incorpo- } \\
\text { rates external variables, indirect and } \\
\text { direct determinants }\end{array}$ & Adults with type 2 diabetes & Adapted \\
\hline $\begin{array}{l}\text { Newman- } \\
\text { Casey et al } \\
(2013)^{67}\end{array}$ & $\begin{array}{l}\text { Model of glaucoma } \\
\text { medication adherence }\end{array}$ & $\begin{array}{l}\text { - A conceptual model of medication } \\
\text { adherence that includes components of } \\
\text { knowledge, barriers to adherence and } \\
\text { perceived stress }\end{array}$ & Glaucoma & New \\
\hline $\begin{array}{l}\text { Paterson et al } \\
(20 \mid 8)^{4 I}\end{array}$ & $\begin{array}{l}\text { Model of adherence by } \\
\text { renal transplant recipients }\end{array}$ & $\begin{array}{l}\text { - A model that examines the impact of } \\
\text { sociodemographics, neurocognitive } \\
\text { abilities, depressive symptoms, self-effi- } \\
\text { cacy and problem solving on medication } \\
\text { adherence }\end{array}$ & Renal transplant recipients & New \\
\hline $\begin{array}{l}\text { Piette et al } \\
(2006)^{68}\end{array}$ & $\begin{array}{l}\text { Framework for } \\
\text { understanding chronically } \\
\text { ill patients' medication cost } \\
\text { problems }\end{array}$ & $\begin{array}{l}\text { - A framework consisting of factors that } \\
\text { impact cost-related medication non- } \\
\text { adherence }\end{array}$ & Chronically ill patients & New \\
\hline $\begin{array}{l}\text { Roe et al } \\
(2009)^{52}\end{array}$ & $\begin{array}{l}\text { Process of choosing not to } \\
\text { take medication }\end{array}$ & $\begin{array}{l}\text { - A model of the steps involved in the } \\
\text { decision to stop taking medication }\end{array}$ & Serious mental illness & New \\
\hline $\begin{array}{l}\text { Schafheutle } \\
\text { et al }(2018)^{54}\end{array}$ & Medication work & $\begin{array}{l}\text { - A framework describing types of medi- } \\
\text { cation work that patients with chronic } \\
\text { obstructive pulmonary disease use post- } \\
\text { discharge }\end{array}$ & $\begin{array}{l}\text { Chronic obstructive pulmonary } \\
\text { disease }\end{array}$ & $\begin{array}{l}\text { Applied } \\
\text { Cheraghi-Sohi } \\
\text { et al's medication } \\
\text { work framework }\end{array}$ \\
\hline $\begin{array}{l}\text { Schrijvers } \\
\text { et al }(20 \mid 4)^{55}\end{array}$ & $\begin{array}{l}\text { Model of adherence to } \\
\text { prophylaxis in hemophilia }\end{array}$ & $\begin{array}{l}\text { - A model to describe factors impacting } \\
\text { adherence to prophylaxis }\end{array}$ & Haemophilia & New \\
\hline $\begin{array}{l}\text { Simoni et al } \\
(2006)^{43}\end{array}$ & $\begin{array}{l}\text { Model of social support } \\
\text { and adherence }\end{array}$ & $\begin{array}{l}\text { - A cognitive-affective model of adher- } \\
\text { ence to highly active antiretroviral } \\
\text { therapy and the impact of social support }\end{array}$ & $\begin{array}{l}\text { HIV-Positive Men and Women } \\
\text { on Antiretroviral Therapy }\end{array}$ & New \\
\hline $\begin{array}{l}\text { Swanlund } \\
\text { et al }(2008)^{56}\end{array}$ & $\begin{array}{l}\text { Medication self- } \\
\text { management model }\end{array}$ & $\begin{array}{l}\text { - A model for predicting one's self-man- } \\
\text { agement capability and the overall suc- } \\
\text { cessful or unsuccessful self-management } \\
\text { of a medication regimen }\end{array}$ & $\begin{array}{l}\text { Community-dwelling older } \\
\text { adults }\end{array}$ & New \\
\hline $\begin{array}{l}\text { Weaver et al } \\
(2005)^{44}\end{array}$ & $\begin{array}{l}\text { Stress and coping model of } \\
\text { medication adherence }\end{array}$ & $\begin{array}{l}\text { - A model of latent factors and indicators } \\
\text { of latent factors that affect adherence to } \\
\text { highly active antiretroviral therapy }\end{array}$ & Adults with HIV & New \\
\hline $\begin{array}{l}\text { Saslo } \\
(2007)^{53}\end{array}$ & $\begin{array}{l}\text { Saslo's model for } \\
\text { adherence to HIV } \\
\text { treatment }\end{array}$ & $\begin{array}{l}\text { - A model of HIV treatment adherence } \\
\text { that includes themes related to fear, } \\
\text { trust, self-worth, finances, literacy and } \\
\text { disease }\end{array}$ & Adults with HIV & New \\
\hline $\begin{array}{l}\text { Qu et al } \\
(2018)^{42}\end{array}$ & $\begin{array}{l}\text { Information motivation } \\
\text { behaviour skills model of } \\
\text { medication adherence } \\
\text { behavior }\end{array}$ & $\begin{array}{l}\text { - A model that examines the relationship } \\
\text { between knowledge, behaviour and } \\
\text { motivation and their impact on adher- } \\
\text { ence rate }\end{array}$ & $\begin{array}{l}\text { Men who have sex with men (at } \\
\text { risk of contracting HIV) }\end{array}$ & Adapted \\
\hline
\end{tabular}

(Continued) 
Table 2 (Continued).

\begin{tabular}{|c|c|c|c|c|}
\hline $\begin{array}{l}\text { Author } \\
\text { (Year) }\end{array}$ & $\begin{array}{l}\text { Name of Framework } / \\
\text { Model }\end{array}$ & Description & Target Population & $\begin{array}{l}\text { New or } \\
\text { Adapted } \\
\text { Framework }\end{array}$ \\
\hline $\begin{array}{l}\text { O'Brien } \\
(2014)^{59}\end{array}$ & Conceptual framework & $\begin{array}{l}\text { - A framework that incorporates contex- } \\
\text { tual factors (risk and protective) and } \\
\text { self-management processes to examine } \\
\text { their effect on medication adherence }\end{array}$ & Older adults & Adapted \\
\hline $\begin{array}{l}\text { Mondesir } \\
\text { et al }(2019)^{51}\end{array}$ & $\begin{array}{l}\text { World Health } \\
\text { Organization } \\
\text { multidimensional } \\
\text { adherence model }\end{array}$ & $\begin{array}{l}\text { - A model based on the five factors of the } \\
\text { World Health Organization's adherence } \\
\text { model }\end{array}$ & $\begin{array}{l}\text { Adults with coronary heart } \\
\text { disease }\end{array}$ & $\begin{array}{l}\text { Applied the } \\
\text { World Health } \\
\text { Organization } \\
\text { Multidimensional } \\
\text { Adherence } \\
\text { Model }\end{array}$ \\
\hline $\begin{array}{l}\text { Messina et al } \\
(2016)^{40}\end{array}$ & $\begin{array}{l}3 \text { Models: } \\
\text { I. Non-medical use of pain } \\
\text { relievers } \\
\text { 2. Non-medical use of } \\
\text { tranquillizers and sedatives } \\
\text { 3. Non-medical use of } \\
\text { stimulants }\end{array}$ & $\begin{array}{l}\text { - Three models that incorporate latent } \\
\text { constructs impacting the non-medical } \\
\text { use of pain relievers, tranquilizers and } \\
\text { sedatives and stimulants }\end{array}$ & Undergraduate students & New \\
\hline $\begin{array}{l}\text { MacDonell } \\
\text { et al }(2016)^{39}\end{array}$ & $\begin{array}{l}\text { Model of medication } \\
\text { adherence }\end{array}$ & $\begin{array}{l}\text { - A model of medication adherence that } \\
\text { incorporates social and cognitive factors }\end{array}$ & $\begin{array}{l}\text { Ethnic and Racial Minority HIV- } \\
\text { Positive Youth }\end{array}$ & New \\
\hline $\begin{array}{l}\text { Kekwaletswe } \\
\text { et al }(2017)^{37}\end{array}$ & $\begin{array}{l}\text { Structural equation model } \\
\text { among HIV positive people } \\
\text { taking antiretroviral } \\
\text { therapy }\end{array}$ & $\begin{array}{l}\text { - A model of latent and measured vari- } \\
\text { ables on adherence to antiretroviral } \\
\text { therapy }\end{array}$ & $\begin{array}{l}\text { HIV positive people taking } \\
\text { antiretroviral therapy }\end{array}$ & Adapted \\
\hline $\begin{array}{l}\text { Dehn } \\
(2010)^{33}\end{array}$ & $\begin{array}{l}\text { Adapted health belief } \\
\text { model }\end{array}$ & $\begin{array}{l}\text { - An adapted model to examine the rela- } \\
\text { tionship between sociodemographic } \\
\text { characteristics, modifying factors and } \\
\text { treatment adherence }\end{array}$ & Adults with cystic fibrosis & Adapted \\
\hline
\end{tabular}

Abbreviations: NR, not reported; HIV, human immunodeficiency virus.

complexity/factors (eg number and size of pills, frequency of refills). There were no major differences between the framework domains from quantitative and qualitative studies.

\section{Discussion}

This scoping review analyzed the range and nature of research on medication self-management frameworks. Within the three self-management domains (ie, medical, emotional and role management), the vast majority focused on medical management (eg adherence, medication-taking behaviour, medication self-efficacy, relationships with healthcare providers), with a limited focus on emotional and role management. Further, within medical management, the majority of studies focused specifically on medication adherence and lacked a comprehensive, holistic conceptualization of self-management. Given the prevalence of individuals taking medications and the potential for problematic polypharmacy, ${ }^{7,69}$ it is important that research and clinical practice conceptualizes medication management beyond adherence to promote person-centred care.

While limited, there were a few frameworks that included a more holistic approach to medication selfmanagement. ${ }^{50,56,62}$ For example, Blalock used behavioural science theories (eg social cognitive theory, theory of social support, Health Belief Model) to describe how to improve medication use among individuals requiring pharmacotherapy. ${ }^{62}$ An ecological model was used to 
present factors impacting medication use at five levels characteristics of the patient; services from providers; support from family, friends and small groups; characteristics of the community and health care system; and social policy and government regulations. Blalock then proposed a framework specific to medication self-management that built on the ecological model and Fisher's self-management model ${ }^{70}$ to incorporate specific intervention goals at each level. The author highlighted the value of using this as a guiding framework for medication self-management, but also identified the need to further develop it as knowledge evolves.

A more holistic framework for medication self-management was also presented by Mickelson et al. ${ }^{50}$ A qualitative study was conducted among older adults with heart failure to describe the medication management work process (effort and time to produce or accomplish something) and adapt Unertl et al's Workflow Elements Model. ${ }^{71}$ The adapted model contained five main process, each containing subprocesses: sensemaking (information gathering, adapting mental models, story building), planning (generating action plans, adapting plans, anticipatory thinking), monitoring (problem detection, tracking), decision making (applying rules, pattern matching, mental stimulation, making trade-offs) and coordinating (reconciling information, managing interdependencies, negotiating). Furthermore, these frameworks highlight the intrinsic complexities of medication management that are critical to consider when developing programs, interventions and initiatives targeting medication self-management.

Importantly, medication-taking has a substantial impact on an individual's life. ${ }^{72}$ For example, a systematic review was conducted by Mohammed et al to explore medication burden among patients with experience taking medications. ${ }^{72}$ This review identified patients' experiences with medication-related burden (ie, burden caused by medication routines, medication characteristics, adverse events, healthcare system, social life), medication-related beliefs (individual attitudes, coping skills, outside influences) and medication-taking practices (following instructions, accepting medication use, modifying care plans). Further, Mohammed et al's review highlighted the impact of medications and medication burden on the day-to-day lives of individuals taking medications, as it interfered with their daily activities and influenced their beliefs, attitudes and overall quality of life. The impact of medication management on an individual's life extends beyond medical management to include emotional and role management. Therefore, it is critical for medication selfmanagement frameworks to be all-encompassing of the self-management domains.

Frameworks that took into account the participants' sociodemographic and clinical characteristics are lacking based on our scoping review. Medication self-management may be impacted by micro, meso and macro level factors. Micro, or individual, level factors can impact medication use and the potential for polypharmacy, as age, gender, cognitive and physical ability and multimorbidity have been noted in the literature to affect medication-taking behaviour. $^{73-78}$ For example, a systematic review was conducted by Smaje et al (2018) to identify factors associated with medication adherence in older patients and found that older age, multimorbidity, cognitive impairment and being male were negatively associated with adherence. ${ }^{74}$ Additionally, characteristics of a medication regimen, including complexity, dose, frequency and side effects can also impact medication adherence. ${ }^{79-83}$ Increased regimen complexity results in reduced medication adherence, as identified in a systematic review by Pantuzza et al (2017) that examined evidence on the association between medication regimen complexity and adherence. $^{79}$ Similarly, a qualitative study conducted by O'Donovan et al (2019) in the United Kingdom explored the impact and management of medication side effects among individuals 18 or older who had experienced side effects. $^{84}$ It was identified that participants used nonadherent behaviours as a method of coping with side effects. Further, many of these individual level factors have also been identified as having an impact on general self-management, ${ }^{85-87}$ which adds a layer of complexity to medication self-management.

Macro or system level factors can also have an impact on medication self-management. This review identified that, to date, the majority of frameworks for medication self-management were from the United States $(n=24)$, with an overall lack of global representation. Medication selfmanagement may look different in different regions or countries based on healthcare system and funding structures, insurance coverage and support from and access to healthcare providers. ${ }^{88,89}$ In an analysis of health data from 1980 to 2016, Sarnak et al compared drug spending and trends in the United States, Australia, Canada, France, Germany, the Netherlands, Norway, Sweden, Switzerland and the United Kingdom. ${ }^{88}$ The United States had considerably higher prescription drug spending and drug prices than the other high-income countries. Americans were also 
more likely to experience high out-of-pocket costs for medications. High out-of-pocket medication costs can impact medication self-management, as an inability to afford medications may lead to cost-related nonadherence. ${ }^{90}$ It is important to acknowledge contextual factors in the development and application of medication self-management frameworks. In addition, more work is needed to explore what sociodemographic and clinical characteristics, as well as what system level factors impact medication self-management in order to understand if, and how, frameworks can be applied to different populations in different contexts.

\section{Future Work}

This scoping review highlighted several gaps in the literature and areas for future work. First, it is important for future research to develop a framework for medication self-management that directly incorporates patients' and providers' voices (ie, through co-design) and can be applied to a broader population of individuals taking medications. Integrating the thoughts, experiences, beliefs and concerns of persons with lived experience and providers is important, as patients are the ones selfmanaging and impacted by these processes on a day-today basis and providers are supporting patients with their health conditions and medications. More specifically, co-design would allow for the collaborative development of a framework that meets the needs of patients and providers. Second, a framework that encompasses all components of medication and self-management should be developed and validated. Current frameworks mostly focus on adherence, rather than incorporating the impact of experiences, beliefs and preferences on selfmanagement behaviour. Frameworks guide research and consequently clinical practice recommendations. ${ }^{91,92}$ Advancing the collective knowledge on medication self-management can help patients and providers navigate self-management and related self-management support.

\section{Limitations}

There are a few limitations of this scoping review that should be noted. First, it is possible that relevant articles were missed based on the databases and grey literature searched. Second, our search was conducted in English, so it is possible that articles published in other languages were not identified. Third, some may note that a critical appraisal of included articles was not undertaken. However, critical appraisal is a less common and optional component for scoping reviews. $^{27}$

\section{Conclusion}

Medication self-management is complex and has the potential to impact multiple aspects of an individual's life, including mental and physical well-being, as well as day-to-day activities. The majority of frameworks included in this review focus on medical management, with few incorporating components of emotional and role management. It is important to acknowledge the impact medication self-management can have on all aspects of one's life and focus future work on developing and validating holistic frameworks for medication self-management that can be applied to a broad population.

\section{Data Sharing Statement}

All data analyzed in this scoping review are included in this published article and its supplementary files.

\section{Ethics Approval and Informed Consent}

Not applicable.

\section{Acknowledgments}

The authors would like to thank the University of Toronto librarian, Glyneva Bradley-Ridout, for her expertise and assistance in developing the search strategy.

\section{Funding}

Dr Guilcher is supported by a Canadian Institutes of Health Research Embedded Clinician Scientist Salary Award on Transitions in Care. Kadesha James is supported by the Dean's Graduate Scholarship (2019-2021) at the Leslie Dan Faculty of Pharmacy, University of Toronto.

\section{Disclosure}

The authors report no potential competing interests with respect to the research, authorship and/or publication of this article.

\section{References}

1. Chao Y-S, Wu C-J, Wu H-C, Chen W-C, Marengoni A. Drug trends among non-institutionalized Canadians and the impact of data collection changes in the Canadian Health Measures Survey 2007 to 2015. PLoS One. 2019;14(4):4. doi:10.1371/journal.pone.0214718 
2. Feely A, Lix LM, Reimer K. Estimating multimorbidity prevalence with the Canadian Chronic Disease Surveillance System. Res Policy Pract. 2017;37:7.

3. Holloway K, van Dijk L. The World Medicines Situation 2011: Rational Use of Medicine. Geneva: World Health Organization; 2011.

4. Kantor ED, Rehm CD, Haas JS, Chan AT, Giovannucci EL. Trends in prescription drug use among adults in the United States from 1999-2012. JAMA. 2015;314(17):1818-1831.

5. Zhang F, Mamtani R, Scott FI, Goldberg DS, Haynes K, Lewis JD. Increasing use of prescription drugs in the United Kingdom. Pharmacoepidemiol Drug Saf. 2016;25(6):628-636.

6. Masnoon N, Shakib S, Kalisch-Ellett L, Caughey GE. What is polypharmacy? A systematic review of definitions. BMC Geriatr. 2017;17:1.

7. Duerden M, Avery T, Payne R. Polypharmacy and Medicines Optimisation: Making It Safe and Sound. London, United Kingdom: The King's Fund;2013.

8. Lown Institute. Medication Overload: America's Other Drug Problem. How the Drive to Prescribe is Harming Older Adults. Massachusetts, USA: Lown Institute; 2019.

9. Canadian Pharmacists Association. Medication Management. 2017. Available from: https://www.pharmacists.ca/education-practiceresources/professional-development/medication-management/.

Accessed November 6, 2017.

10. Burns A, American Pharmacists Association. Medication therapy management in pharmacy practice: core elements of an MTM service model (version 2.0). J Am Pharm Assoc. 2008;48(3):341-353. doi:10.1331/JAPhA.2008.08514

11. Barlow J, Wright C, Sheasby J, Turner A, Hainsworth J. Self-management approaches for people with chronic conditions: a review. Patient Educ Couns. 2002;48(2):177-187. doi:10.1016/S0738-3991 (02)00032-0

12. Lorig KR, Holman HR. Self-management education: history, definition, outcomes, and mechanisms. Ann Behav Med. 2003;26(1):1-7.

13. Funnell MM, Brown TL, Childs BP, et al. National standards for diabetes self-management education. Diabetes Care. 2008;31 (Supplement 1):S97.

14. Captieux M, Pearce G, Parke HL, et al. Supported self-management for people with type 2 diabetes: a meta-review of quantitative systematic reviews. BMJ Open. 2018;8(12):e024262.

15. Glazier RH, Bajcar J, Kennie NR, Willson K, Systematic A. Review of interventions to improve diabetes care in socially disadvantaged populations. Diabetes Care. 2006;29(7):1675.

16. Greenwood DA, Gee PM, Fatkin KJ, Peeples M, Systematic A. Review of reviews evaluating technology-enabled diabetes self-management education and support. J Diabetes Sci Technol. 2017;11(5):1015-1027.

17. Mohamed A, Staite E, Ismail K, Winkley K. A systematic review of diabetes self-management education interventions for people with type 2 diabetes mellitus in the Asian Western Pacific (AWP) region. Nurs Open. 2019;6(4):1424-1437.

18. Audulv Å, Ghahari S, Kephart G, Warner G, Packer TL. The Taxonomy of Everyday Self-management Strategies (TEDSS): a framework derived from the literature and refined using empirical data. Patient Educ Couns. 2019;102(2):367-375.

19. McGreal MH, Hogan MJ, Walsh-Irwin C, Maggio NJ, Jurgens CY. Heart failure self-care interventions to reduce clinical events and symptom burden. Res Rep Clin Cardiol. 2014;5:243-257.

20. Jonkman Nini H, Westland H, Groenwold Rolf HH, et al. Do selfmanagement interventions work in patients with heart failure? Circulation. 2016;133(12):1189-1198.

21. Pinnock H, Parke HL, Panagioti M, et al. Systematic meta-review of supported self-management for asthma: a healthcare perspective. BMC Med. 2017;15(1):64.

22. Hodkinson A, Bower P, Grigoroglou C, et al. Self-management interventions to reduce healthcare use and improve quality of life among patients with asthma: systematic review and network metaanalysis. BMJ. 2020;370:m2521.
23. van Grieken RA, van Tricht MJ, Koeter MWJ, van den Brink W, Schene AH. The use and helpfulness of self-management strategies for depression: the experiences of patients. PLoS One. 2018;13(10): e0206262-e0206262.

24. Arksey H, O’Malley L. Scoping studies: towards a methodological framework. Int J Soc Res Methodol. 2005;8(1):19-32.

25. Levac D, Colquhoun H, O'Brien KK. Scoping studies: advancing the methodology. Implement Sci. 2010;5:69.

26. Peters MDJ, Marnie C, Tricco AC, et al. Updated methodological guidance for the conduct of scoping reviews. JBI Evidence Synthesis. 2020;18(10):2119-2126.

27. Tricco AC, Lillie E, Zarin W, et al. Prisma extension for scoping reviews (PRISMA-ScR): checklist and explanation. Ann Intern Med. 2018;169(7):467-473.

28. Bramer WM, Giustini D, de Jonge GB, Holland L, Bekhuis T. Deduplication of database search results for systematic reviews in EndNote. J Med Libr Assoc. 2016;104(3):240-243.

29. McHugh ML. Interrater reliability: the kappa statistic. Biochemia Medica. 2012;22(3):276-282.

30. Archiopoli A, Ginossar T, Wilcox B, Avila M, Hill R, Oetzel J. Factors of interpersonal communication and behavioral health on medication self-efficacy and medication adherence. AIDS Care. 2016;28(12):1607-1614.

31. Bright EE, Stanton AL. Prospective investigation of social support, coping, and depressive symptoms: a model of adherence to endocrine therapy among women with breast cancer. J Consult Clin Psychol. 2018;86(3):242-253.

32. de Guzman AB, Guevara KIJ, Guiang FJB, et al. Developing a model of medication adherence among Filipino elderly. Educ Gerontol. 2013;39(5):298-313.

33. Dehn CP Coping styles and physician partnership in adults with cystic fibrosis: relation to treatment adherence. 2010;Ph.D.:162 p-162.

34. Dilorio C, Shafer PO, Letz R, et al. Project EASE: a study to test a psychosocial model of epilepsy medication management. Epilepsy Behav. 2004;5(6):926-936.

35. Fransen GAJ, Mesters I, Janssen MJR, Knottnerus JA, Muris JWM. Which patient-related factors determine self-perceived patient adherence to prescribed dyspepsia medication? Health Educ Res. 2009;24 (5):788-798.

36. Guy AA, Niel K, Du Bois SN. Emotional support, active coping, and ART adherence in young African American men who have sex with men: a pilot mediation model. J Assoc Nurs AIDS Care. 2018;29 (4):612-618.

37. Kekwaletswe CT, Jordaan E, Nkosi S, Morojele NK. Social support and the mediating roles of alcohol use and adherence self-efficacy on Antiretroviral Therapy (ART) adherence among ART recipients in Gauteng, South Africa. AIDS Behav. 2017;21(7):1846-1856.

38. Lau Y, Htun TP, Chan KS, Klainin-Yobas P. Multidimensional factors affecting medication adherence among community-dwelling older adults: a structural-equation-modeling approach. J Public Health (Germany). 2017;25(1):113-122.

39. MacDonell KK, Jacques-Tiura AJ, Naar S, Fernandez MI. Predictors of self-reported adherence to antiretroviral medication in a multisite study of ethnic and racial minority HIV-positive youth. $J$ Pediatr Psychol. 2016;41(4):419-428.

40. Messina BG, Dutta NM, Silvestri MM, et al. Modeling motivations for non-medical use of prescription drugs. Addict Behav. 2016;52:46-51.

41. Paterson TSE, O'Rourke N, Jean Shapiro R, Thornton WL. Medication adherence in renal transplant recipients: a latent variable model of psychosocial and neurocognitive predictors. PLoS One. 2018;13(9):e0204219.

42. Qu D, Zhong X, Xiao G, Dai J, Liang H, Huang A. Adherence to preexposure prophylaxis among men who have sex with men: a prospective cohort study. Int J Infect Dis. 2018;75:52-59. 
43. Simoni JM, Frick PA, Huang B. A longitudinal evaluation of a social support model of medication adherence among HIV-positive men and women on antiretroviral therapy. Health Psychol. 2006;25(1):74-81.

44. Weaver KE, Llabre MM, Duran RE, et al. A stress and coping model of medication adherence and viral load in HIV-positive men and women on highly active antiretroviral therapy (HAART). Health Psychol. 2005;24(4):385-392.

45. Beusterien KM, Davis EA, Flood R, Howard K, Jordan J. HIV patient insight on adhering to medication: a qualitative analysis. AIDS Care. 2008;20(2):244-252.

46. Brown TM, Siu K, Walker D, Pladevall-Vila M, Sander S, Mordin M. Development of a conceptual model of adherence to oral anticoagulants to reduce risk of stroke in patients with atrial fibrillation. $J$ Manag Care Pharm. 2012;18(5):351-362.

47. Coleman A. Medication adherence of elderly citizens in retirement homes through a mobile phone adherence monitoring framework (Mpamf) for developing countries: a case study in South Africa. Indian J Pharm Educ Res. 2014;48(3):6-11.

48. Ghose T, Ali S, Shubert V, Stanton M, Walker L, Chaudhuri S. "It's my Room and my Life": housing's Influence on Medication Adherence for HIV-positive Women Released from Incarceration. $J$ Health Care Poor Underserved. 2019;30(1):182-201.

49. Koh JJK, Cheng RX, Yap Y, et al. Access and adherence to medications for the primary and secondary prevention of atherosclerotic cardiovascular disease in Singapore: a qualitative study. Patient Prefer Adherence. 2018;12:2481-2498.

50. Mickelson RS, Unertl KM, Holden RJ. Medication management: the macrocognitive workflow of older adults with heart failure. JMIR Human Factors. 2016;3(2):e27.

51. Mondesir FL, Levitan EB, Malla G, et al. Patient perspectives on factors influencing medication adherence among people with Coronary Heart Disease (CHD) and CHD risk factors. Patient Prefer Adherence. 2019;13:2017-2027.

52. Roe D, Goldblatt H, Baloush-Klienman V, Swarbrick M, Davidson L. Why and how people decide to stop taking prescribed psychiatric medication: exploring the subjective process of choice. Psychiatr Rehabil J. 2009;33(1):38-46.

53. Saslo MC Ethnography of adherence to plans of care in the HIV positive patient. 2007;D.N.S.:112 p-112 p.

54. Schafheutle EI, Fegan T, Ashcroft DM. Exploring medicines management by COPD patients and their social networks after hospital discharge. Int J Clin Pharm. 2018;40(5):1019-1029.

55. Schrijvers L, Kars M, Beijlevelt-van Der Zande M, Peters M, Schuurmans M, Fischer K. Perspectives of patients with hemophilia on adherence to prophylaxis: a grounded theory approach. Haemophilia. 2014;20(SUPPL. 3):118.

56. Swanlund SL, Scherck KA, Metcalfe SA, Jesek-Hale SR. Keys to successful self-management of medications. Nurs Sci Q. 2008;21 (3):238-246

57. Corneli A, Perry B, McKenna K, et al. Participants' explanations for nonadherence in the FEM-PrEP clinical trial. J Acquir Immune Defic Syndr. 2016;71(4):452-461.

58. Nantha YS, Haque S, Nantha HS, Swarna nantha Y, Swarna Nantha H. The development of an integrated behavioural model of patient compliance with diabetes medication: a mixed-method study protocol. Fam Pract. 2019;36(5):581-586.

59. O'Brien A-M Factors associated with medication adherence in frail urban older adults: a descriptive and explanatory study. 2014; $\mathrm{Ph}$. D.:180 p-180 p.

60. Alsalman AJ, Smith WR. Expanding the framework of assessing adherence and medication-taking behavior. J Pain Palliat Care Pharmacother. 2013;27(2):114-124.

61. Bartlett Ellis RJ, Welch JL. Medication-taking behaviours in chronic kidney disease with multiple chronic conditions: a meta-ethnographic synthesis of qualitative studies. J Clin Nurs. 2017;26(5-6):586-598.
62. Blalock SJ. The theoretical basis for practice-relevant medication use research: patient-centered/behavioral theories. Res Social Admin Pharm. 2011;7(4):317-329.

63. Gifford AL, Groessl EJ. Chronic disease self-management and adherence to HIV medications. J Acquir Immune Defic Syndr. 2002;31 (SUPPL. 3):S163-S166.

64. Goh H, Kwan YH, Seah Y, Low LL, Fong W, Thumboo J. A systematic review of the barriers affecting medication adherence in patients with rheumatic diseases. Rheumatol Int. 2017;37(10):16191628.

65. Li WW, Stotts NA, Froelicher ES. Compliance with antihypertensive medication in Chinese immigrants: cultural specific issues and theoretical application. Res Theory Nurs Pract. 2007;21(4):236-254.

66. McCann TV, Clark E, Lu S. The self-efficacy model of medication adherence in chronic mental illness. J Clin Nurs. 2008;17(11):329340 .

67. Newman-Casey PA, Weizer JS, Heisler M, Lee PP, Stein JD. Systematic review of educational interventions to improve glaucoma medication adherence. Semin Ophthalmol. 2013;28(3):191-201.

68. Piette JD, Heisler M, Horne R, Caleb Alexander G. A conceptually based approach to understanding chronically ill patients' responses to medication cost pressures. Soc Sci Med. 2006;62(4):846-857.

69. Gallagher P, Lang PO, Cherubini A, et al. Prevalence of potentially inappropriate prescribing in an acutely ill population of older patients admitted to six European hospitals. Eur J Clin Pharmacol. 2011;67 (11):1175-1188.

70. Fisher EB, Brownson CA, O'Toole ML, Shetty G, Anwuri VV, Glasgow RE. Ecological approaches to self-management: the case of diabetes. Am J Public Health. 2005;95(9):1523-1535.

71. Unertl KM, Novak LL, Johnson KB, Lorenzi NM. Traversing the many paths of workflow research: developing a conceptual framework of workflow terminology through a systematic literature review. J Am Med Inform Assoc. 2010;17(3):265-273.

72. Mohammed MA, Moles RJ, Chen TF. Medication-related burden and patients' lived experience with medicine: a systematic review and metasynthesis of qualitative studies. BMJ Open. 2016;6(2):e010035.

73. Shruthi R, Jyothi R, Pundarikaksha HP, Nagesh GN, Tushar TJA. Study of medication compliance in geriatric patients with chronic illnesses at a tertiary care hospital. J Clin Diagn Res. 2016;10(12): FC40-FC43.

74. Smaje A, Weston-Clark M, Raj R, Orlu M, Davis D, Rawle M. Factors associated with medication adherence in older patients: a systematic review. AGING MEDICINE. 2018;1(3):254-266.

75. Turner A, Hochschild A, Burnett J, Zulfiqar A, Dyer CB. High prevalence of medication non-adherence in a sample of communitydwelling older adults with adult protective services-validated selfneglect. Drugs Aging. 2012;29(9):741-749.

76. Cárdenas-Valladolid J, Martín-Madrazo C, Salinero-Fort MA, et al. Prevalence of adherence to treatment in homebound elderly people in primary health care. Drugs Aging. 2010;27(8):641-651.

77. Altıparmak S, Altıparmak O. Drug-using behaviors of the elderly living in nursing homes and community-dwellings in Manisa, Turkey. Arch Gerontol Geriatr. 2012;54(2):e242-e248.

78. Manteuffel M, Williams S, Chen W, Verbrugge RR, Pittman DG, Steinkellner A. Influence of patient sex and gender on medication use, adherence, and prescribing alignment with guidelines. $J$ Women's Health. 2014;23(2):112-119.

79. Pantuzza LL, Ceccato M, Silveira MR, Junqueira LMR, Reis AMM. Association between medication regimen complexity and pharmacotherapy adherence: a systematic review. Eur J Clin Pharmacol. 2017;73(11):1475-1489.

80. Ayele AA, Tegegn HG, Ayele TA, Ayalew MB. Medication regimen complexity and its impact on medication adherence and glycemic control among patients with type 2 diabetes mellitus in an Ethiopian general hospital. BMJ Open Diabetes Res Care. 2019;7(1):e000685. 
81. Coleman CI, Limone B, Sobieraj DM, et al. Dosing frequency and medication adherence in chronic disease. J Manag Care Pharm. 2012;18(7):527-539.

82. Dibonaventura M, Gabriel S, Dupclay L, Gupta S, Kim E. A patient perspective of the impact of medication side effects on adherence: results of a cross-sectional nationwide survey of patients with schizophrenia. BMC Psychiatry. 2012;12:20.

83. Milan R, Vasiliadis H-M. The association between side effects and adherence to antidepressants among primary care community-dwelling older adults. Aging Ment Health. 2020;24(8):1229-1236.

84. O’Donovan B, Rodgers RM, Cox AR, Krska J. 'You feel like you haven't got any control': a qualitative study of side effects from medicines. J Patient Saf Risk Manage. 2019;24(1):13-24.

85. Cramm JM, Twisk J, Nieboer AP. Self-management abilities and frailty are important for healthy aging among community-dwelling older people; a cross-sectional study. BMC Geriatr. 2014;14(1):28.

86. Mathew R, Gucciardi E, De Melo M, Barata P. Self-management experiences among men and women with type 2 diabetes mellitus: a qualitative analysis. BMC Fam Pract. 2012;13(1):122.

87. Shao J-H, Chen S-H. Who did it better? Gender differences in effects of a dietary self-management intervention for older communitydwelling adults. $J$ Women Aging. 2019;1-14.
88. Sarnak DO, Squires D, Kuzmak G, Bishop S. Paying for Prescription Drugs Around the World: why Is the U.S. an Outlier? Issue Brief. 2017;2017:1-14.

89. Canada's Premiers. The pan-Canadian Pharmaceutical Alliance. 2020; https:/www.canadaspremiers.ca/pan-canadian-pharmaceuticalalliance-archives/.

90. Gupta S, McColl MA, Guilcher SJ, Smith K. Cost-related nonadherence to prescription medications in Canada: a scoping review. Patient Prefer Adherence. 2018;12:1699-1715.

91. American Academy of Family Physicians. Clinical Practice Guideline Manual. 2017. Available from: https://www.aafp.org/ family-physician/patient-care/clinical-recommendations/cpg-manual. html\#: :text=Definition $\% 3 \mathrm{~A} \% 20$ Clinical\%20practice $\% 20$ guidelines $\%$ 20are,harms $\% 20$ of $\% 20$ alternative $\% 20$ care $\% 20$ options. Accessed May 7, 2021.

92. Rycroft-Malone J, Bucknall T. Using theory and frameworks to facilitate the implementation of evidence into practice. Worldviews Evid Based Nurs. 2010;7(2):57-58.
Patient Preference and Adherence

\section{Publish your work in this journal}

Patient Preference and Adherence is an international, peer-reviewed, open access journal that focusing on the growing importance of patient preference and adherence throughout the therapeutic continuum. Patient satisfaction, acceptability, quality of life, compliance, persistence and their role in developing new therapeutic modalities and compounds to optimize clinical outcomes for existing disease

\section{Dovepress}

states are major areas of interest for the journal. This journal has been accepted for indexing on PubMed Central. The manuscript management system is completely online and includes a very quick and fair peer-review system, which is all easy to use. Visit http:// www.dovepress.com/testimonials.php to read real quotes from published authors. 